\title{
Proteins associated with pancreatic cancer survival in patients with resectable pancreatic ductal adenocarcinoma
}

\author{
Ru Chen ${ }^{1}$, David W Dawson ${ }^{2,3}$, Sheng Pan ${ }^{1}$, Niki A Ottenhof ${ }^{4}$, Roeland F de Wilde ${ }^{4}$, Christopher L Wolfgang ${ }^{5}$, \\ Damon H May ${ }^{6}$, David A Crispin ${ }^{1}$, Lisa A Lai ${ }^{1}$, Anna R Lay ${ }^{2}$, Meghna Waghray ${ }^{7}$, Shouli Wang ${ }^{8}$, Martin W Mclntosh ${ }^{6}$, \\ Diane M Simeone ${ }^{7}$, Anirban Maitra ${ }^{9}$ and Teresa A Brentnall ${ }^{1}$
}

Pancreatic ductal adenocarcinoma (PDAC) is a highly lethal disease with a dismal prognosis. However, while most patients die within the first year of diagnosis, very rarely, a few patients can survive for $>10$ years. Better understanding the molecular characteristics of the pancreatic adenocarcinomas from these very-long-term survivors (VLTS) may provide clues for personalized medicine and improve current pancreatic cancer treatment. To extend our previous investigation, we examined the proteomes of individual pancreas tumor tissues from a group of VLTS patients (survival $\geq 10$ years) and short-term survival patients (STS, survival $<14$ months). With a given analytical sensitivity, the protein profile of each pancreatic tumor tissue was compared to reveal the proteome alterations that may be associated with pancreatic cancer survival. Pathway analysis of the differential proteins identified suggested that MYC, IGF1R and p53 were the top three upstream regulators for the STS-associated proteins, and VEGFA, APOE and TGF $\beta$ - 1 were the top three upstream regulators for the VLTS-associated proteins. Immunohistochemistry analysis using an independent cohort of 145 PDAC confirmed that the higher abundance of ribosomal protein S8 (RPS8) and prolargin (PRELP) were correlated with STS and VLTS, respectively. Multivariate Cox analysis indicated that 'High-RPS8 and Low-PRELP' was significantly associated with shorter survival time $(\mathrm{HR}=2.69,95 \% \mathrm{Cl} 1.46-4.92, P=0.001)$. In addition, galectin-1, a previously identified protein with its abundance aversely associated with pancreatic cancer survival, was further evaluated for its significance in cancerassociated fibroblasts. Knockdown of galectin-1 in pancreatic cancer-associated fibroblasts dramatically reduced cell migration and invasion. The results from our study suggested that PRELP, LGALS1 and RPS8 might be significant prognostic factors, and RPS8 and LGALS1 could be potential therapeutic targets to improve pancreatic cancer survival if further validated.

Laboratory Investigation (2015) 95, 43-55; doi:10.1038/labinvest.2014.128; published online 27 October 2014

The prognosis of pancreatic ductal adenocarcinoma (PDAC) is extremely poor: the majority of patients die within the 6 months of diagnosis, and only $6 \%$ of patients survive for 5 years. ${ }^{1}$ At the time of diagnosis, most patients have surgically unresectable disease. For the patients who undergo surgical resection, the clinical outcome is considerably better, with 5year survival rates of up to $20 \%$ in some studies..$^{2-11}$ In very rare situations, some patients can survive for a very long term (very-long-term survivors (VLTS), defined as patients with $\geq 10$-year survival following resection) and are essentially cured of their disease. There are some published studies that have identified VLTS of histologically validated PDAC, usually at a frequency $<5 \%$ in most series. ${ }^{2,3,10,12}$ Surprisingly, patients with aggressive diseases such as T3-T4 lesions and involvement of lymph node were also presented in these VLTS cohorts, suggesting that the basis of VLTS in PDAC extends beyond simply resecting node-negative localized cases but might indicate an intrinsic molecular phenotype of such tumors. ${ }^{13}$

\footnotetext{
${ }^{1}$ Department of Medicine, University of Washington, Seattle, WA, USA; ${ }^{2}$ Department of Pathology and Laboratory Medicine, UCLA, Los Angeles, CA, USA; ${ }^{3}$ Jonsson Comprehensive Cancer Center, David Geffen School of Medicine, UCLA, Los Angeles, CA, USA; ${ }^{4}$ Department of Pathology, Sol Goldman Pancreatic Cancer Research Center, The Johns Hopkins Medical Institutions, Baltimore, MD, USA; ${ }^{5}$ Department of Surgery, Sol Goldman Pancreatic Cancer Research Center, The Johns Hopkins Medical Institutions, Baltimore, MD, USA; ${ }^{6}$ Fred Hutchinson Cancer Research Center, Molecular Diagnostics Program, Seattle, WA, USA; ${ }^{7}$ Department of Surgery, University of Michigan, Ann Arbor, MI, USA; ${ }^{8}$ Department of Pathology, Soochow University School of Medicine, Suzhou, China and ${ }^{9}$ Department of Surgical Oncology, The University of Texas MD Anderson Cancer Center, Houston, TX, USA 
Several clinicopathological characteristics have been demonstrated to have significant effect on long-term survival, including: small tumor size $(<2 \mathrm{~cm})$, negative lymph node status, negative resection margin (ie, R0), and differentiation grade. ${ }^{14}$ However, the molecular characteristics relating to long-term survival of PDAC are poorly understood. Using a PDAC survival tissue microarray (TMA), a study evaluated 13 putative biomarkers selected from the literature and identified mucin 1 and mesothelin as biomarkers for predicting aggressiveness of pancreatic cancer biology, outperforming pathological features and other putative biomarkers. ${ }^{15}$ In our previous efforts, using pooled samples we discovered that the abundance of stromal galectin-1 (LGALS1) was adversely associated with survival from PDAC, while the abundance of prolargin (PRELP), osteoglycin (OGN) and rheumatoid factors D5 light chain (Vк3) were higher in the pancreatic tumor tissues of VLTS relative to short-term survival (STS). ${ }^{13}$ Immunohistochemistry (IHC) analysis of pancreatic tumor tissues using two independent cohorts confirmed that an increased/strong abundance of galectin-1, a T-cell immune-modulator and metastasis-related protein, in the cancer stroma was a negative indicator of pancreatic cancer survival. ${ }^{13}$ For PRELP, contradictorily, a recent study suggested that the high abundance of PRELP was associated with shorter pancreatic cancer survival. ${ }^{16}$ Although various factors could influence the observations in different studies, the role of PRELP in pancreatic cancer survival remains uncertain and warrants further investigation.

In the current study, we sought to expand our understanding of the proteome characteristics associated with pancreatic cancer survival by analyzing the individual cancerous tissues from VLTS and STS patients using a label-free proteomics approach. Functional protein annotation and pathway analysis of the differential proteins associated with VLTS and STS aided in the characterization of the mechanisms underlying survival. Although a comprehensive interpretation of the data obtained will require additional information beyond this investigation, selected exploration of a few interesting proteins are presented here. IHC validation was performed using a clinically annotated TMA to examine the significance of the protein targets in the context of PDAC survival. In addition, a functional study was performed to evaluate the effects of galectin- 1 on the metastatic behavior of cancer-associated fibroblasts (CAFs).

\section{METHODS AND MATERIALS}

\section{Patients and Specimens}

This study was approved by the Human Subjects Division of the University of Washington, the Internal Review Board (IRB) of the University of California at UCLA and the IRB of the Johns Hopkins Medical Institutions. VLTS and STS were identified as survivors of PDAC for $\geq 10$ years and $<14$ months, respectively, following surgical resection. Before inclusion, the histology was reviewed for all of the identified VLTS and STS cases from Johns Hopkins by two experts in pancreatic pathology (RHH and AM), in order to confirm the diagnosis of PDAC and exclude the possibility of confounding lesions, such as ampullary cancers and/or intraductal papillary mucinous neoplasm. Five VLTS and five STS cases were matched by tumor stage and grade for proteomic analysis. These tumors were obtained prior to treatment, and thus the proteomes represented the naive state of the adenocarcinoma. The changing standards for adjuvant chemotherapy over the past 15 years precluded us from matching cases by type of treatment received. The validation cohort was from UCLA with 145 PDAC cases constructed on a previously described TMA. ${ }^{17}$ The clinicopathological and survival information for each patient were obtained from UCLA surgical database of pancreatic patients. Survival analysis of the UCLA cohort was limited to overall survival, which was determined by review of clinical records and search of the Social Security Death Index. Survival intervals were calculated from the date of surgery to the date of confirmed death or last patient contact. The clinicopathological and survival information of the PDAC cases included in our study are provided in Table 1.

\section{Protein Extraction from Formalin-Fixed Paraffin-Embedded (FFPE) Tissue}

VLTS of pancreatic cancer is rare, and the availability of fresh frozen tissue specimens from these VLTS patients is therefore extremely scarce. The current study made use of the commonly preserved format of clinical specimens (ie, FFPE) for comparative proteomics analysis. ${ }^{18}$ Extraction of proteins from FFPE tissue were carried out as described in our previous study. ${ }^{18}$ Briefly, unstained $15-\mu \mathrm{m}$ sections of FFPE tissue were deparaffinized and rehydrated. The corresponding H\&E slides were examined under the microscope to delineate the areas with the highest neoplastic cellularity (including both PDAC epithelium and associated stroma), while excluding obvious areas of non-neoplastic pancreatic acinar tissues and inflammatory infiltrates. An equivalent of $1 \mathrm{~cm} \times 1 \mathrm{~cm} \times 15 \mu \mathrm{m}$ amount of deparaffinized tissue samples were scraped from each slide and transferred into $300 \mu \mathrm{l}$ lysis solution of $70 \% 50 \mathrm{mM}$ ammonium bicarbonate and $30 \%$ acetonitrile, using sterile blades and needle tips. The lysates were then incubated at $90{ }^{\circ} \mathrm{C}$ for $30 \mathrm{~min}$ followed by $60{ }^{\circ} \mathrm{C}$ for $120 \mathrm{~min}$. The samples were sonicated for $2 \mathrm{~min}$ followed by 1-min incubation on ice. The sonication and ice incubation process was repeated twice more. The homogenized samples were incubated at $60^{\circ} \mathrm{C}$ for $1 \mathrm{~h}$, followed by a second sonication step.

\section{Sample Preparation for Proteomics Analysis}

The protein lysates were digested with sequencing-grade trypsin (Promega, Madison, WI, USA) at a trypsin-toprotein ratio of $1: 50$ at $37^{\circ} \mathrm{C}$ for $18 \mathrm{~h}$. The digested samples were centrifuged at $1500 \mathrm{~g}$ for $10 \mathrm{~min}$, and the supernatants were collected. The protein digests were reduced with $10 \mathrm{mM}$ dithiothreitol at $60^{\circ} \mathrm{C}$ for $60 \mathrm{~min}$ and then incubated with 
Table 1 Clinicopathological and survival information for the PDAC cases

\begin{tabular}{lccc}
\hline Cohort & VLST & STS & UCLA TMA cohort \\
\hline $\begin{array}{l}\text { No. of cases } \\
\text { Age, years }\end{array}$ & 5 & 5 & 145 \\
$\quad<60$ & 2 & 1 & 49 \\
$\geq 60$ & 3 & 4 & 96 \\
& & & \\
Gender & 3 & 2 & 75 \\
M & 2 & 3 & 70 \\
F & & & \\
& & & \\
Survival time & & & \\
Mean & $>10$ years & 10 months & 34.7 months \\
Range & $>10$ years & $6-14$ months & $0.49-100$ months
\end{tabular}

$$
\begin{gathered}
\text { Tumor size } \\
<3 \mathrm{~cm}
\end{gathered}
$$$$
\geq 3 \mathrm{~cm}
$$

Tumor grade

Low grade (G1-2)

High grade (G3-4)

AJCC stage
1
2
Unknown

Lymph nodes

Negative

Positive

1
4
0

0

5
$25 \mathrm{mM}$ iodoacetamide at room temperature in the dark for $30 \mathrm{~min}$ for cysteine alkylation. The samples were then purified with C18 columns (UltraMicroSpin Column/Vydac C18 silica, The Nest Group, Southborough, MA, USA), dried down and stored at $-20{ }^{\circ} \mathrm{C}$ until mass spectrometric analysis.

\section{LC MS/MS Analysis}

An LTQ-Orbitrap hybrid mass spectrometer (Thermo Fisher Scientific, Waltham, MA, USA) coupled with a nano-flow HPLC (Eksigent Technologies, Dublin, CA, USA) was used in this study. A 2- $\mu \mathrm{g}$ sample of each case was injected for the mass spectrometric analysis. The samples were first loaded onto a $1.5-\mathrm{cm}$ trap column (IntegraFrit $100 \mu \mathrm{m}$, New Objective, Woburn, MA, USA) packed with Magic C18AQ resin ( $5 \mu \mathrm{m}, 200 \AA$ particles; Michrom Bioresources, Auburn, CA, USA) with Buffer A (water with $0.1 \%$ formic acid) at a flow rate of $3 \mu \mathrm{l} / \mathrm{minute}$. The peptide samples were then separated by an $27-\mathrm{cm}$ analytical column (PicoFrit $75 \mu \mathrm{m}$, New Objective) packed with Magic C18AQ resin $(5 \mu \mathrm{m}, 100 \AA$ particles; Michrom Bioresources) followed by mass spectrometric analysis. A 90-min linear LC gradient was used as follows: $5-7 \%$ Buffer B (acetonitrile with $0.1 \%$ formic acid) vs Buffer A over $2 \mathrm{~min}$, then to $35 \%$ over $90 \mathrm{~min}$, then to $50 \%$ over $1 \mathrm{~min}$, hold at $50 \%$ for $9 \mathrm{~min}$, change to $95 \%$ over $1 \mathrm{~min}$, hold at $95 \%$ for $5 \mathrm{~min}$, drop to $5 \%$ over $1 \mathrm{~min}$, and recondition at $5 \%$. The flow rate for the peptide separation was $300 \mathrm{nl} /$ minute. For MS analysis, a spray voltage of $2.25 \mathrm{kV}$ was applied to the nanospray tip. The mass spectrometry experiment was performed using data-dependent acquisition with a $\mathrm{m} / \mathrm{z}$ range of 400-1800, consisting of a full MS scan in the Orbitrap followed by up to five MS/MS spectra acquisitions in the linear ion trap using collisioninduced dissociation. Other mass spectrometer parameters include: isolation width $2 \mathrm{~m} / \mathrm{z}$, target value 1e4, collision energy $35 \%$, max injection time $100 \mathrm{~ms}$. Lower abundance peptide ions were interrogated using dynamic exclusion (exclusion time $45 \mathrm{~s}$, exclusion mass width $-0.55 \mathrm{~m} / z$ low to $1.55 \mathrm{~m} / \mathrm{z}$ high). A charge state screen was used, allowing for MS/MS of any ions with identifiable charge states $+2,+3$ and +4 and higher. The samples were analyzed in duplicate with random order using the identical instrument conditions.

\section{Proteomics Data Analysis}

Raw machine output files from all MS runs were converted to mzXML files and searched with X!Tandem ${ }^{19}$ configured with the $k$-score scoring algorithm, ${ }^{20}$ against version 3.69 of the human International Protein Index database. The search parameters were as follows: enzyme, trypsin; maximum missed cleavages, 1; fixed modification, carboxamidomethylation on cysteine; potential modification, oxidization on methionine; parent monoisotopic mass error, 2.5 Da. Peptide identifications were assigned probability by PeptideProphet, ${ }^{21}$ and all identifications with an assigned probability $<0.95$ were discarded (estimated false discovery rate (FDR) varied per experimental run in the range 0.006-0.007). Protein inference were assigned by ProteinProphet ${ }^{22}$ using the peptides with probability scores $\geq 0.95$. Any proteins with only one spectrum identified in one group and none in the other group were excluded from ratio calculation and subsequent analysis. The comparative analysis between the VLTS and STS was achieved using spectral counts of all the peptides in each protein/protein group. Both replicate data were included in the data analysis as averaging the spectral counts from the duplicate experiments reduces the technical variations. The protein ratio between the VLTS group and STS group was calculated using the total spectral counts of five VLTS samples divided by the total spectral count of five STS samples. 


\section{Functional Annotation, Enrichment and Pathway Analysis}

The enrichment of the biological processes was assessed by using The Database for Annotation, Visualization and Integrated Discovery (DAVID ) v6.7. ${ }^{23}$ The pathway analysis was performed using ingenuity pathways analysis (IPA, Ingenuity Systems). The proteins that had a minimum of two-fold abundance change between the VLTS and STS groups were uploaded to IPA to identify upstream regulators significant to the data set.

\section{IHC Analysis}

FFPE TMA block was sectioned at $4 \mu \mathrm{m}$ onto charged slides, deparaffinized and processed for heat-induced epitope retrieval in $0.01 \mathrm{~mol} / \mathrm{l}$ citric acid buffer, $\mathrm{pH} 6.0$ for $15 \mathrm{~min}$ in a vegetable steamer, followed by incubation with primary antibody against prolargin (Epitomics) or ribosomal protein S8 (RPS8; Proteintech) with 1:100 dilution incubation at room temperature for $1 \mathrm{~h}$. The LSAB + HRP System (Dako, Carpinteria, California, USA) was used for visualization. Three separate $1.0 \mathrm{~mm}$ cores for each annotated tumor in the TMA were independently scored by two blinded observers using semiquantitative histoscores (range 0-300). Histoscores were the product of staining intensity $(0-3)$ and the percentage of tumor cells staining at that intensity (0-100). If any core's histoscore differed by $>30$ points between observers, a revised score was assigned by consensus evaluation. Median histoscore of both observers was used for analysis.

\section{Cell Culture and Stable Transfection}

Human CAF cell line was prepared by the outgrowth method. Fresh tissue was obtained from residual pancreatic adenocarcinoma specimen from one patient undergoing primary surgical resection at the University of Michigan. The human sample was obtained in accordance with the policies and practices of the Institutional Review Board of the University of Michigan. Briefly, tumor sample was minced and seeded in six-well plates containing 15\% FBS/DMEM, L-glutamine $(2 \mathrm{mmol} / \mathrm{l})$, penicillin/streptomycin and amphotericin. After 5 days, cells were able to grow out from the tissue clumps. Medium was changed every 3 days. Cells were maintained at $37^{\circ} \mathrm{C}$ in a humidified atmosphere of $5 \% \mathrm{CO}_{2}$. When fibroblast cells grew to confluence, cells were trypsinized and passaged. The identification of fibroblast cell was determined by IHC for $\alpha$ SMA, vimentin, PDGFR and FAP. The contamination of immunocytes and tumor cells were excluded by CD45 and CK19, respectively.

Galectin-1 knockdown cells were generated by stable transfection of galectin-1 shRNA (Qiagen no. KH19360P) using the Attractene Transfection Reagent (Qiagen) and selection with puromycin as per the manufacturer's suggested protocol. Knockdown was confirmed by immunofluorescence and western blotting analysis using antigalectin-1 antibody (R\&D AF1152) as per the manufacturer's instructions. GAPDH was used as a loading control for western blottings.

\section{Wound Healing and Invasion Assays}

For wound healing, cells were grown to confluence on glass coverslips. Cells were manually wounded with a pipet tip. Twenty-four hours later, cells were fixed and mounted onto slides with Prolong Gold plus DAPI (Invitrogen). Images were taken with a Leica DMLB microscope equipped with a Diagnostic Instruments Color Mosaic camera and software. Invasion assays were performed as described. ${ }^{24}$

\section{Statistical Analysis}

All statistics were performed using SPSS, version 20.0, for Windows. $\chi^{2}$ tests were used to compare dichotomized IHC groups against baseline clinicopathological factors. Univariate Cox regression was performed to evaluate the prognostic significance of individual variables. Kaplan-Meier survival curves were analyzed by log-rank test. Multivariate analysis was performed by stepwise Cox analysis with backward selection using the Akaike Information Criterion. The level of significance for all tests was defined as $\alpha=0.05$.

\section{RESULTS}

\section{Proteomics Analysis}

Five pathologically and clinically well-characterized samples from each group of VLTS and STS were included and analyzed in duplicate. The samples were interrogated in a random order using the same reversed-phase analytical column, LC gradient and mass spectrometer parameters. Using only the peptides identified with a PeptideProphet probability score $\geq 0.95 \quad(\mathrm{FDR}<1 \%), 1050$ non-redundant proteins (based on gene symbol) was identified. To evaluate the reproducibility of the platform, all 10 samples analyzed were combined to assess the correlation of peptide intensity between two replicate runs. The ion intensity of the peptides in each analysis was plotted $v s$ each other. A high correlation of the peptide intensities between the replicates was observed (Spearman's $R=0.95$; Supplementary Figure $S 1$ ), indicating a robust technical reproducibility.

\section{Proteins Associated with Pancreatic Cancer Survival}

For each protein identified, the protein ratio between VLTS and STS were quantified by calculating the ratio of total spectral counts between all five VLTS cases and all five STS cases. The spectral count information can be found in Supplementary Table S1. For the proteins that were detected in VLTS group, but not in STS group, the ratio of VLTS vs STS was assigned 100; and for the proteins that were only detected in STS group, the ratio of VLTS vs STS was assigned 0.01 . Using two-fold ratio as a threshold, 332 differentially expressed proteins between VLTS and STS were identified, including 139 proteins with $\geq 2$-fold increase in abundance in the VLTS group (designated as 'proteins associated with the VLTS group'), and 193 proteins with $\geq 2$-fold abundant 
increase in the STS group (designated as 'proteins associated with the STS group'). Figure 1 shows the distribution of protein ratios (natural log-transformed) calculated between the VLTS and STS groups, excluding the proteins with a ratio with 0.01 or 100 . Notably, the differentially expressed proteins identified in this study are relative between VLTS and STS, and many of them were found frequently overexpressed in pancreatic tumor tissues when compared with normal pancreas. ${ }^{18,25-27}$

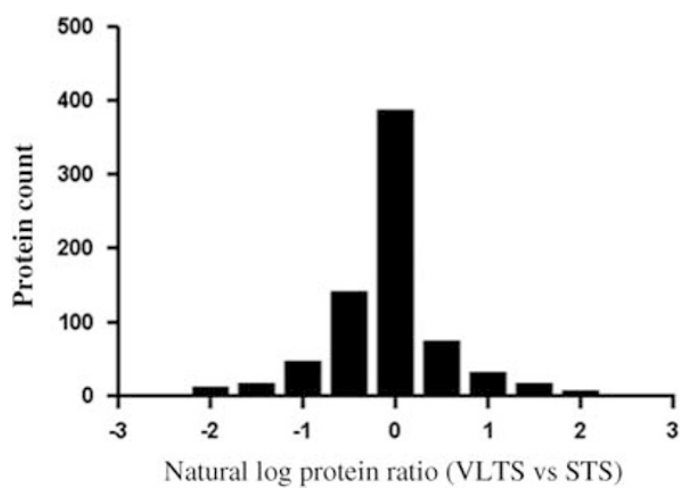

Figure 1 Protein ratio histogram. Distribution of the protein spectral count ratios (VLTS/STS in natural log scale).

\section{Functional Enrichment and Upstream Regulation Networks}

To further characterize functional clusters of the proteins associated with the PDAC survival groups, we uploaded the VLTS and STS survival-associated proteins onto DAVID online database for enrichment analysis. ${ }^{23,28}$ The enriched protein groups associated with STS and VLTS are summarized in Table 2. For the STS-associated differential proteins, the most significantly enriched protein cluster was cytoskeleton proteins, with 30 cytoskeleton proteins more abundantly associated with the STS group. Proteins in this cluster were involved in cellular movement, cell division, endocytosis, movement of organelles and maintenance of cell shape. The second enriched cluster of the STS-associated proteins was ribonucleoprotein complex/protein biosynthesis, with 31 differential proteins included in this functional cluster. Within this cluster, there were proteins involved in nucleoporins, translational elongation, ribosomal proteins and protein biosynthesis. For the VLTS-associated differential proteins, the most significantly enriched cluster was copine proteins. Copines are $\mathrm{Ca}^{2+}$-dependent phospholipid-binding proteins that are thought to be involved in membrane trafficking in response to intracellular calcium increase. ${ }^{29}$ Eight copine proteins were more abundantly expressed in the VLTS group. The second enriched cluster of the

Table 2 Enrichment analysis of survival-associated proteins

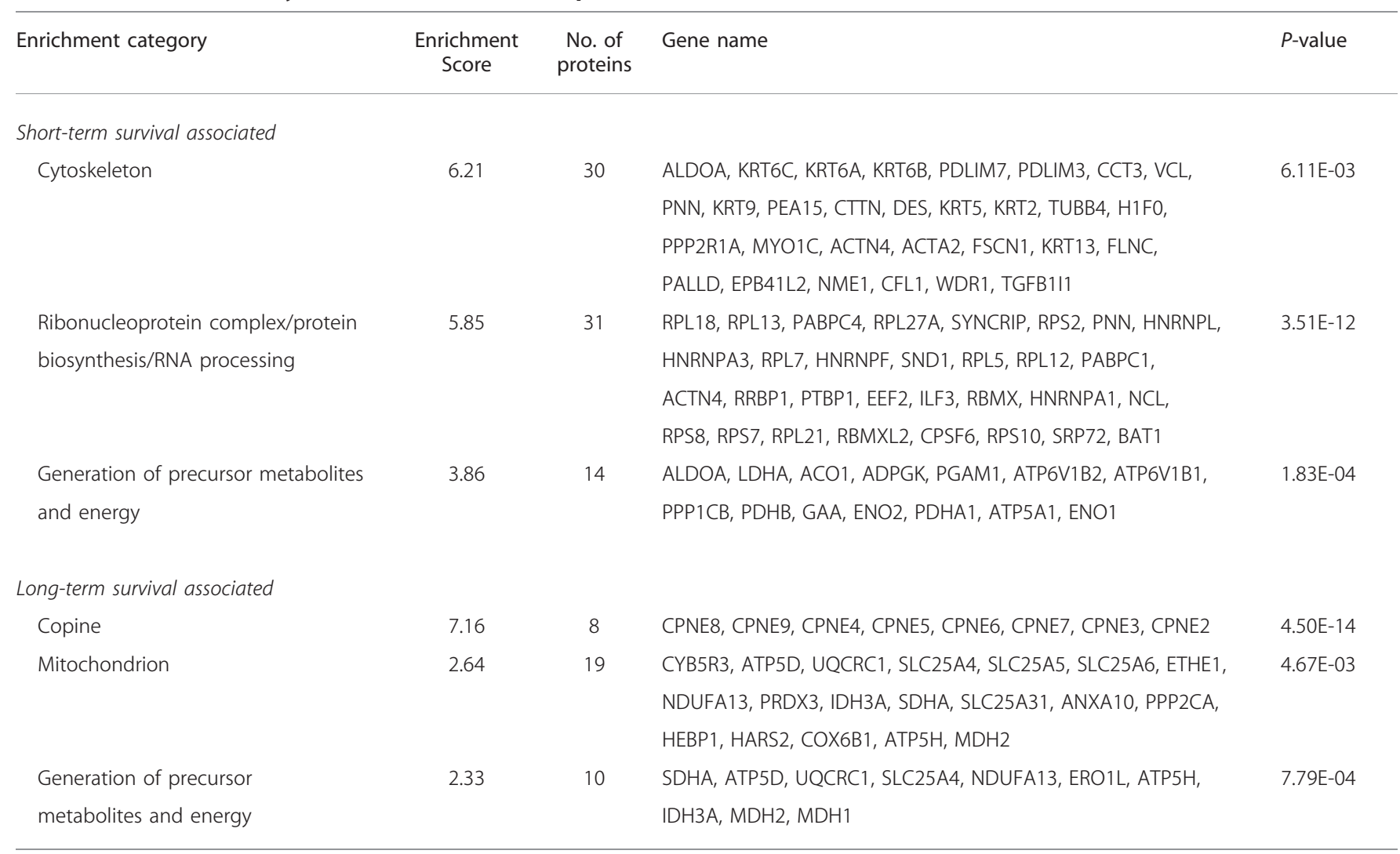


a

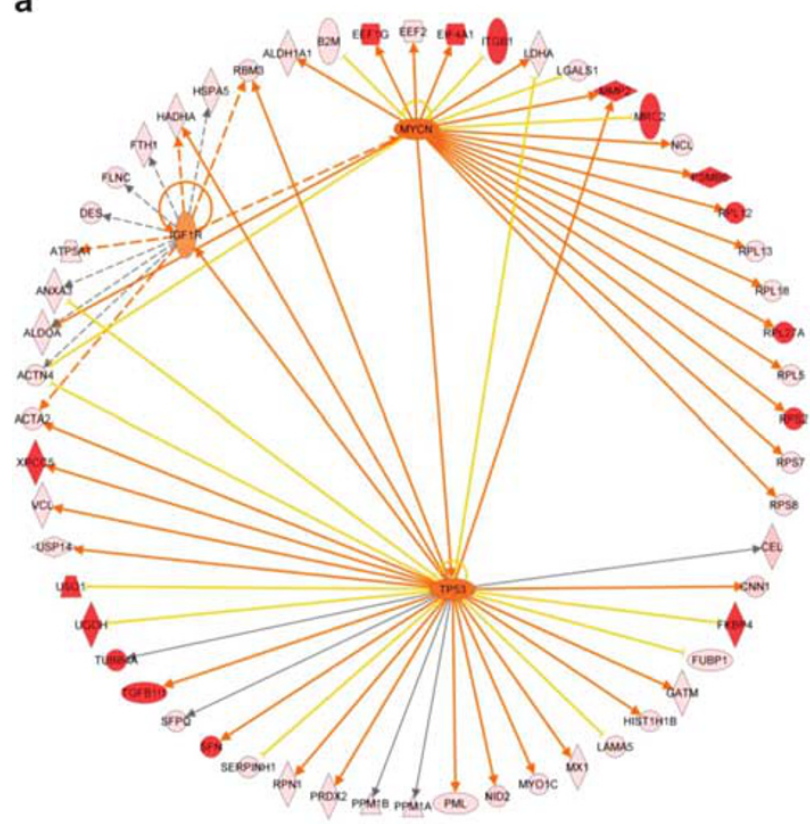

b

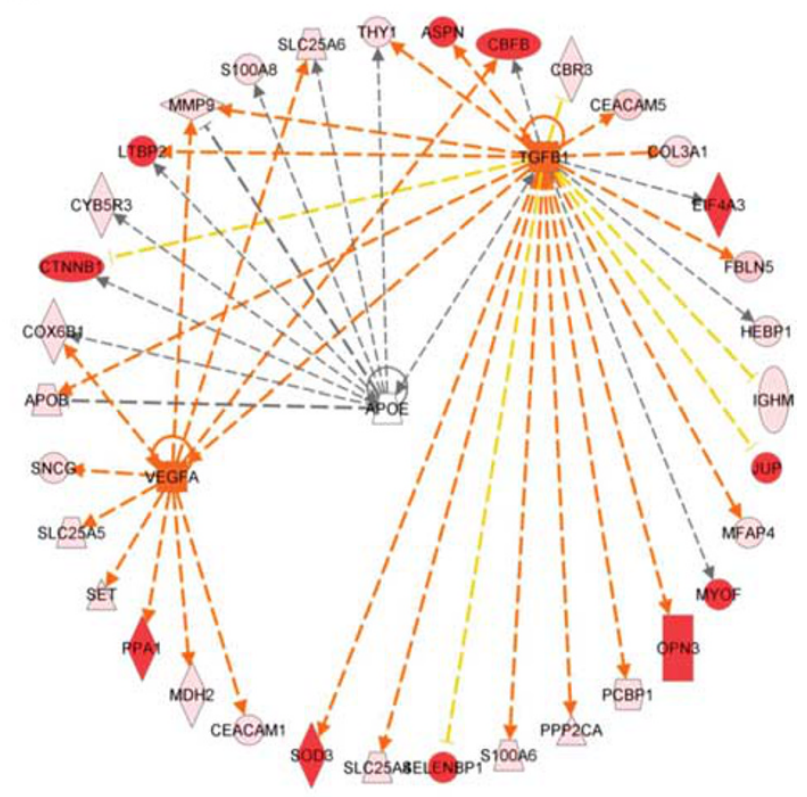

Figure 2 Upstream regulator network in survival-associated proteins. (a) The top three upstream regulators involved in the proteins associated with the STS group: MYC, IGF1R and TP53. (b) The top three upstream regulators involved in the proteins associated with the VLTS group: VEGFA, APOE, TGFB1. Note: Red or pink denotes upregulation in either STS (2A) or VLTS (2B) tumor tissue, orange denotes predicted activation, yellow denotes findings inconsistent with the state of downstream molecule, gray denotes effect not predicted.

VLTS-associated proteins was mitochondrion. Nineteen mitochondrial proteins were more abundantly expressed in the VLTS group, including mitochondrial solute carrier proteins, mitochondrial membrane and matrix proteins and other enzymes involved in cellular respiration. Both the STSand VLTS-associated proteins had an enriched cluster of generation of precursor metabolites and energy. But there were differences within this cluster regarding the survivalrelated proteins that were identified. The 10 VLTS-associated proteins in this cluster were mostly mitochondrial proteins involved in oxidation and reduction process, while only five out of the 14 STS-associated proteins were mitochondrial.

\section{Upstream Regulation Network}

To reveal potential pathways involved in pancreatic cancer survival, IPA was used for upstream regulator analysis. The top three upstream regulators for the STS-associated proteins were MYC, insulin-like growth factor 1 receptor (IGF1R) and TP53 (Figure 2a). MYC is a transcription factor that controls the expression of numerous genes that govern cell proliferation, cell growth, cell differentiation and apoptosis. In our previous tissue proteomics studies, c-MYC was identified as a significant regulator implicated in pancreatic cancer, chronic pancreatitis, as well as precancerous PanIN3. ${ }^{18,25,27}$ In the current study, 25 STS-associated proteins were regulated by MYC. IGF1R has a crucial role in tumor transformation by regulating cell growth and survival. Eleven STS-associated proteins were downstream of IFG1R pathways, five of which were also regulated by MYC. Tumor-suppressor gene p53 is frequently mutated or deleted in pancreatic cancer. The protein product of $\mathrm{p} 53$ gene has a pivotal role monitoring cellular repair and apoptosis in response to genetic damage or metabolite disorder. Twenty-four STS-associated proteins were regulated by TP53 protein. In connection with the other two upstream regulators MYC and IGF1R, the top three upstream regulators and their downstream targets (48 STSassociated proteins) formed a cross-talk network involving two essential traits of cancer cells: proliferation and apoptosis. The top three upstream regulators for VLTS-associated proteins were vascular endothelial growth factor A (VEGFA), apolipoprotein E (APOE) and TGF- $\beta 1$, regulating 10, 9 and 24 VLTS-associated proteins, respectively (Figure $2 \mathrm{~b}$ ). These three upstream regulators linked 35 VLTS-associated proteins in a network that regulated angiogenesis (VEGFA), cholesterol regulation (APOE) and proliferation, differentiation, survival and apoptosis (TGF- $\beta 1$ ). Although the information revealed by IPA is informative and consistent with our understanding of pancreatic tumorigenesis, much work is needed to experimentally investigate these molecular events that can potentially influence pancreatic cancer survival.

\section{Correlation of Prolargin with Longer Pancreatic Cancer Survival}

Prolargin (PRELP) was one of the differential proteins associated with VLTS identified in our previous study. ${ }^{13}$ Based on the current proteomic data, overall, PRELP was 
a

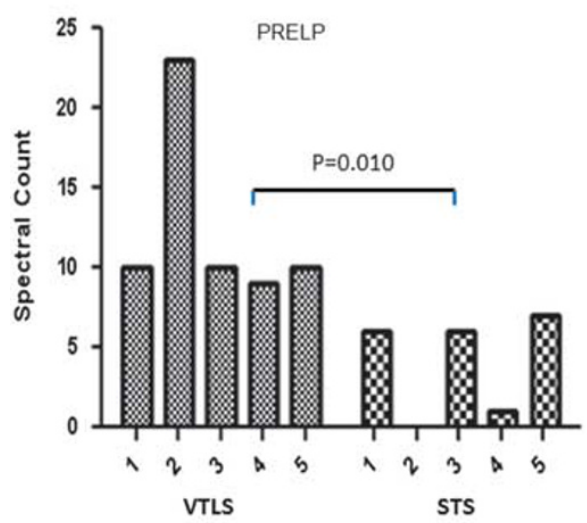

d

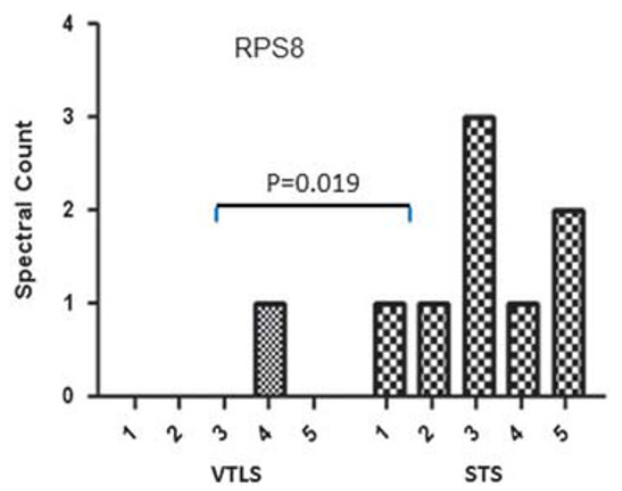

b

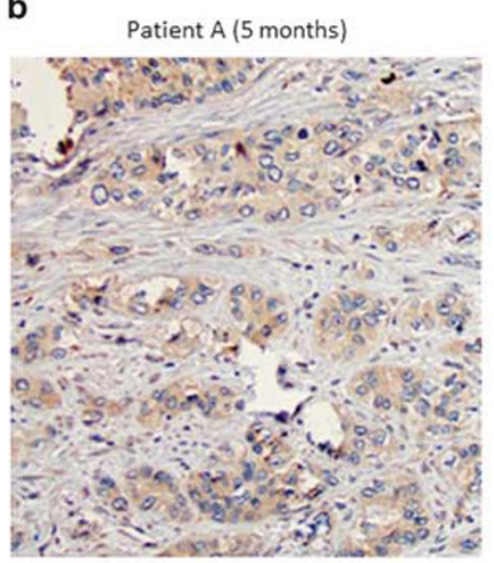

e

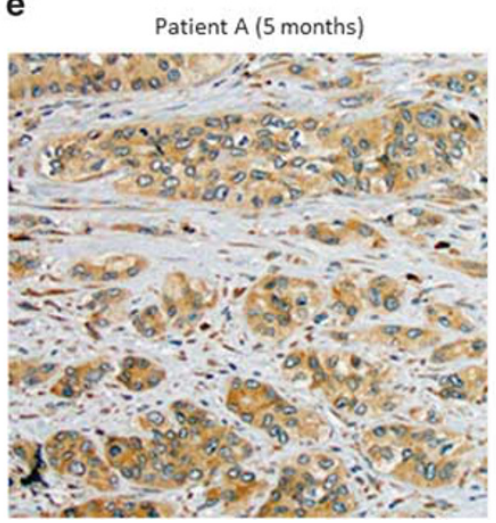

C

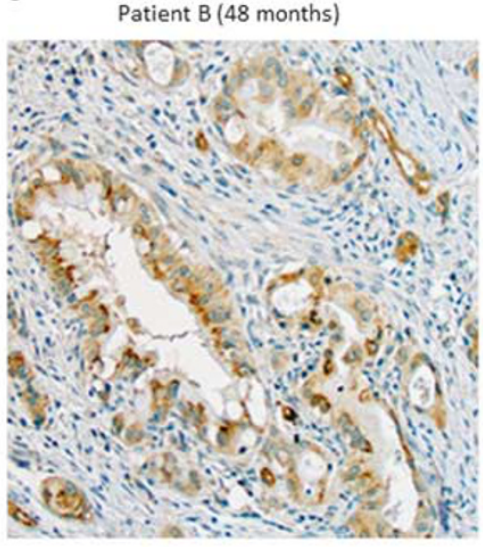

f

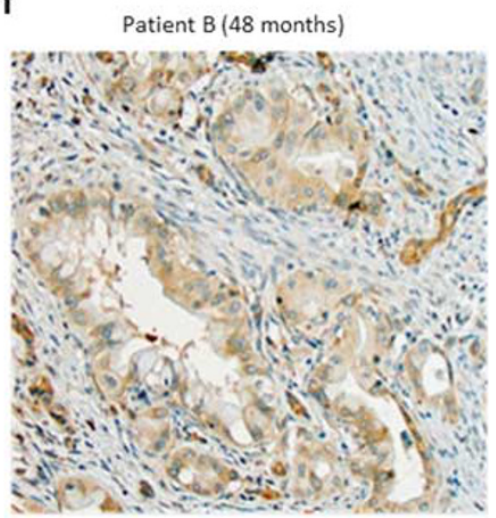

Figure 3 Association of PRELP and RPS8 with survival in PDAC. (a) The expression of PRELP was significantly higher in the tumor tissues of the VLTS group compared to the STS group based on spectral counts. (b and c) Representative IHC staining of PRELP in tumor tissues from a patient with 5 months survival (b) and a patient with 48 months survival (c). (d) The expression of RPS8 was significantly higher in the tumor tissues of the STS group compared with the VLTS group based on spectral counts. (e and f) Representative IHC staining of RPS8 in the tumor tissues from a patient with 5 months survival (e) and a patient with 48 months survival (f). Brown (DAB chromogen) documents positive staining. Images were taken using an x20 objective (200-fold magnification).

3.3-fold more abundant in the tumor tissues of the VLTS group compared with the STS group. The PRELP protein was detectable in all the 5 VLTS cancer samples, with spectral counts ranging from 9 to 23. In contrast, PRELP was marginally detectable in 4 of the 5 STS samples with much lower spectral count number, ranging from 1 to 7 (Figure 3a). The spectral counts of PRELP peptides were significantly higher in the LTS group than in the STS group $(P=0.010)$.

IHC analysis was performed on tissue sections from independent pancreatic cancer samples (UCLA cohort), constructed into a TMA. The UCLA cohort consisted of 145 pancreatic adenocarcinoma patients with a mean survival time of 35 months, ranging from 0.45 to 100 months. Overall, the neoplastic epithelial cells displayed varying degrees of PRELP protein staining, while peritumoral stromal cells were mostly negative with rare weak positivity seen in occasional cases. The IHC scoring was therefore based on tumor cell staining. The survival time of the patients was blinded at the time of IHC scoring. Three out of the 145 specimens were excluded from the analysis because of an absence of tumor cells in the tissue cores available for analysis. The 142 cases were separated into tertiles based on the IHC scores, with top two tertiles being the high PRELP group and the bottom tertile being the low PRELP group. No significant associations were noted between PRELP abundance and baseline clinicopathological parameters, including age, gender, tumor grade, tumor size, tumor stage, lymph node involvement and tumor margin status. Neoplastic cells from tumor tissue of patients with longer survival displayed higher intensity PRELP protein staining than the tumor tissue of patients with shorter survival (Figures $3 b$ and c). Kaplan-Meier survival analysis suggested that patients with high PRELP staining had significantly better survival time $(P=0.013$, Figure 4a). Overall mean survival time for patients with high $v s$ low PRELP staining was 40.9 vs 27.0 months, respectively. Univariate Cox regression analysis confirmed that high abundance of PRELP was significantly associated with improved overall survival (hazard ratio $(\mathrm{HR})=0.61,95 \%$ confidence interval (CI) 0.42-0.91, $P=0.015)$. In the multivariate Cox analysis, high PRELP 



Figure 4 Survival analysis of PRELP and RPS8 immunohistochemistry and patient survival in the validation cohort (UCLA cohort). Patients with higher PRELP staining had better survival (a) and patients with lower RPS8 staining had better survival (b). In the combination analysis of the two types of staining, patients with 'Low RPS8 and High PRELP' had better survival (c) and patient with 'High RPS8 and Low PRELP' had worse survival (d).

abundance fell just short of statistical significance $(\mathrm{HR}=$ $0.68,95 \%$ CI $0.46-1.02$ ) in a model with other significant pathological variables, including high histological grade $(\mathrm{HR}=1.51,95 \% \mathrm{CI} 1.02-2.23)$, positive lymph node involvement $(\mathrm{HR}=1.63,95 \% \mathrm{CI} 1.12-2.39)$ and low pathological T stage $(\mathrm{HR}=0.62,95 \%$ CI 0.41-0.92) (Table $3 \mathrm{a})$.

\section{Correlation of 40 S RPS8 with Shorter Pancreatic Cancer Survival}

Another protein candidate selected for IHC analysis was the 40S RPS8. Overall, RPS8 protein was 7.7-fold more abundant in the tumors from the STS group compared with the VLTS group according to the proteomics data. At the individual sample level, the RPS8 protein was detected in all the five STS tumor samples but in only one VLTS tumor sample (Figure 3d). The spectral counts of RPS8 were significantly higher in STS than in VLST $(P=0.019)$. Moreover, protein network analysis revealed that a large group of ribosomal and ribosomal-associated proteins extensively interact with RPS8 (Supplementary Figure S2). The many proteins from this group were elevated in the tumor tissue of STS patients and highly interactive among themselves, implying that pathways impacting RPS8 and associated ribosomal proteins may have a role in pancreatic cancer survival.

IHC analysis was performed on the TMA of UCLA PDAC cohort, and the scoring was based on tumor cell staining. The 141 scorable cases were separated into quartiles based on the IHC scores, with the top quartile being the high RPS8 group and the bottom three quartiles being the low RPS8 group. No significant associations were noted between RPS8 abundance and baseline clinicopathological parameters as mentioned above. Kaplan-Meier survival analysis suggested that patients with low RPS8 staining had significantly longer survival time $(P=0.011$, Figure $4 \mathrm{~b})$. Representative IHC staining of tumor tissues from patients with longer survival and shorter survival are presented in Figures $3 \mathrm{e}$ and f, respectively. Overall mean survival time for patients with high RPS8 staining was 23.8 compared with 39.3 months for those patients with low RPS8. Univariate Cox regression analysis confirmed that high abundance of RPS8 was significantly associated with poor overall survival $(\mathrm{HR}=1.79,95 \%$ CI $1.13-2.82, P=0.012)$. Multivariate Cox analysis also confirmed that high RPS8 abundance was significantly associated with shorter survival time $(\mathrm{HR}=1.81,95 \%$ CI 1.14-1.2.86) in a model with other 
Table 3 Multivariate COX regression analysis evaluating PRELP and RPS8 staining with PDAC survival

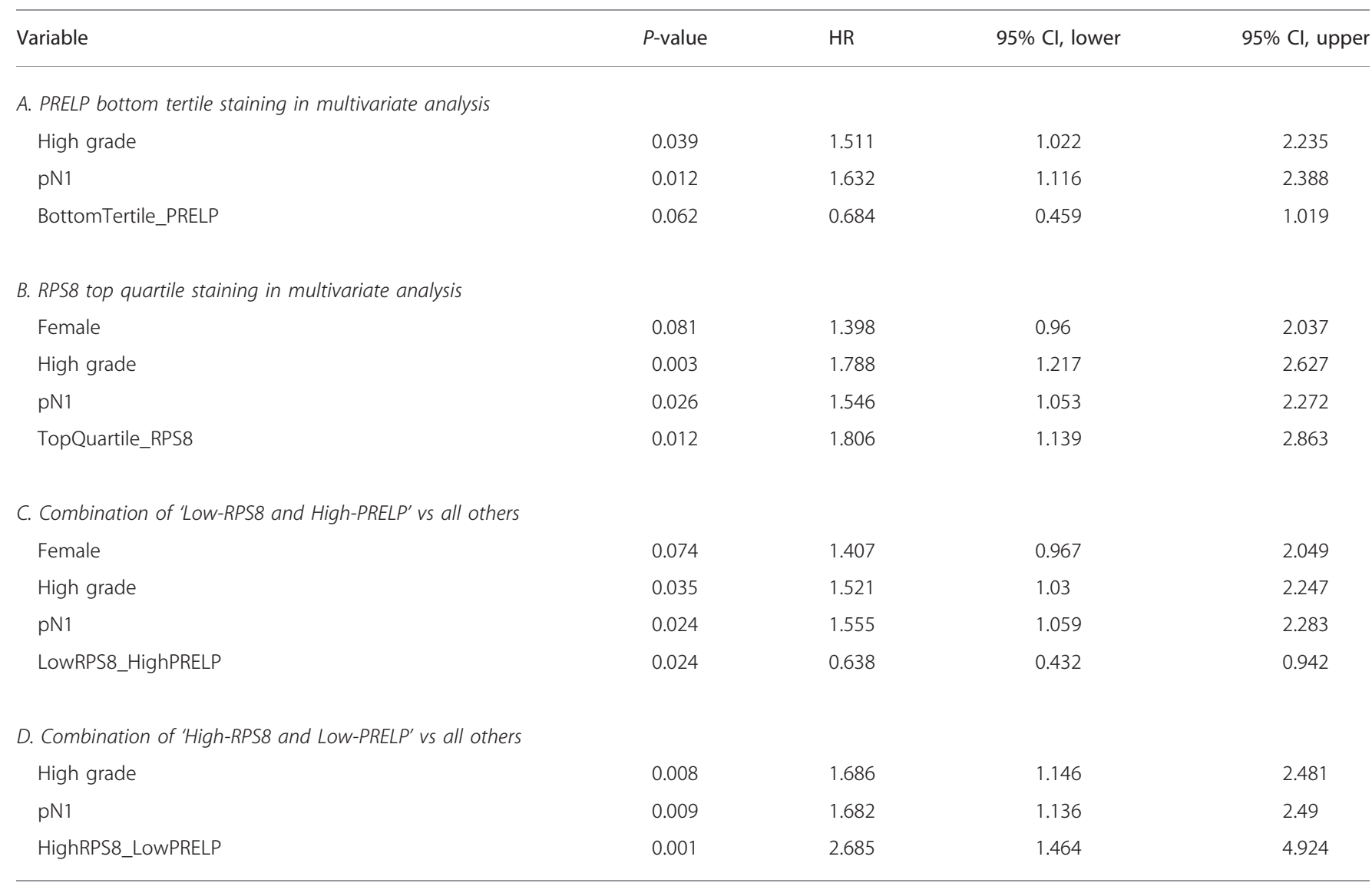

significant pathological variables incorporated, including high histological grade $(\mathrm{HR}=1.79,95 \%$ CI $1.22-2.63)$ and positive lymph node involvement $(\mathrm{HR}=1.55,95 \% \mathrm{CI}$ 1.05-2.27) (Table 3b).

\section{Combination of PRELP and RPS8}

To evaluate the combination of PRELP and PRS8 for survival prediction, we used either 'High-RPS8 and LowPRELP' or 'Low-RPS8 and High-PRELP' for KaplanMeier survival analysis and multivariate Cox analysis. As shown in Figure 4c, 'High-RPS8 and Low-PRELP' was associated with worse survival $(P=0.004)$. Overall mean survival time for cases with 'High-RPS8 and Low-PRELP' status was 16.9 months, while mean survival time for cases with other staining status was 38.0 months. In a separate survival analysis, 'Low-RPS8 and High-PRELP' was associated with markedly improved survival $(P=0.003$, Figure 4d). Overall mean survival time for cases with 'LowRPS8 and High-PRELP' status was 43.1 months, while mean survival time for cases with other staining status was 27.1 months.

Multivariate Cox regression analysis was then used to test the combination of either 'High-RPS8 and Low-PRELP' or 'Low-RPS8 and High-PRELP' along with other variables from baseline clinicopathological parameters. As shown in Table 3(c and d), both 'High-RPS8 and Low-PRELP' and 'Low-RPS8 and High-PRELP' were significantly associated with survival time ( $\mathrm{HR}=2.69$ and 0.64 , respectively). Factors including high histological grade and positive lymph node involvement continued to be significant pathological variables in these multivariate analyses. The combination of 'High-RPS8 and Low-PRELP' displayed improved prognostic value over any single marker alone or the combination of 'Low-RPS8 and High-PRELP'.

\section{Knockdown of Galectin-1 in Pancreatic CAFs Dramatically Reduced Cell Migration and Invasion}

In this study, we observed that galectin-1 was seven-fold higher in the STS group compared with the VLTS group, confirming our previous finding that the abundance of galectin-1 was aversely associated with survival in resectable pancreatic cancer. ${ }^{13}$ As galectin-1 is primarily expressed in CAFs, we sought to determine its functional role in influencing cancer aggressiveness using CAFs. We first generated stable clones of CAFs with galectin-1 knocked down via several distinct galectin-1 shRNA constructs. One of such constructs ( hRNA1) resulted in substantial reduction ( $86 \%$ reduction) of galectin-1 abundance as confirmed by fluorescence immuno- 


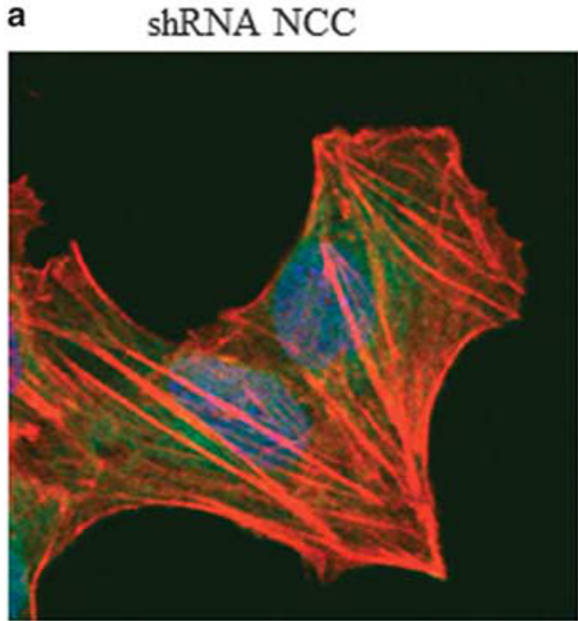

d


b

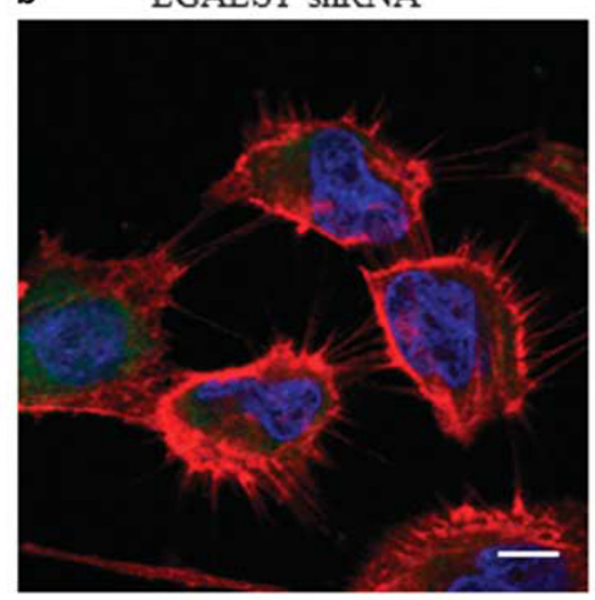

c

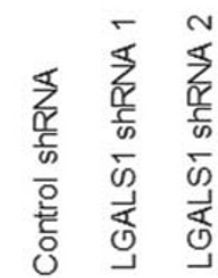

Galectin1

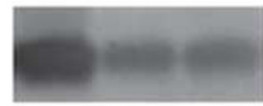

GAPDH



$24 \mathrm{hr}$
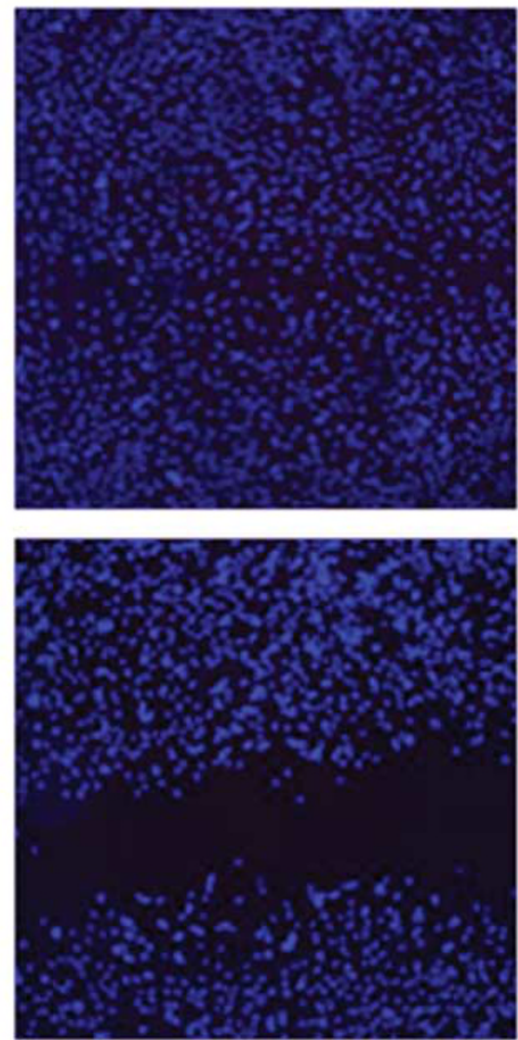

e

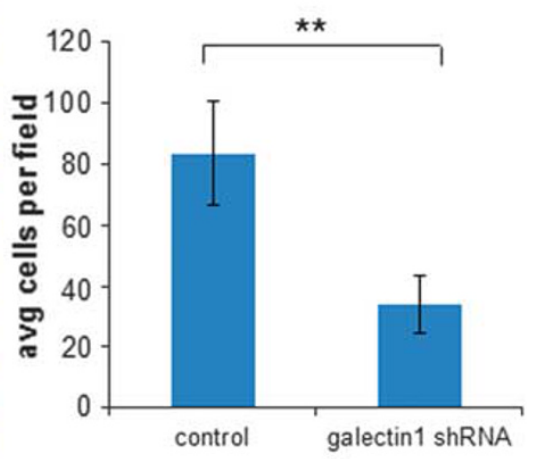

Figure 5 Knockdown of galectin-1 in pancreatic cancer-associated fibroblasts dramatically reduced cell migration and invasion. Shown are representative immunofluorescent images of CAF11-500 cells transfected with control shRNA (NCC, a) or galectin-1 shRNA (b) stained with antigalectin 1 (green), phalloidin (red), and counterstained with DAPI (blue). (c) Western blot analysis showing decreased expression of galectin-1 following transfection with shRNA constructs. GAPDH is shown as a loading control. (d) Wound healing assay shows reduction of cell movement into the wounded area at $24 \mathrm{~h}$ post wounding for galectin-1 knockdown cells (bottom right) compared to control (top right). (e) Invasion assay showed a dramatic reduction in the average number of cells per field for galectin-1 knockdown line compared to control $(t$-test, $P$-value $<0.001)$.

staining and western blotting (Figures $5 \mathrm{a}-\mathrm{c}$ ). In the woundhealing assay, control CAF cells usually closed the wound gap within $24 \mathrm{~h}$ postwounding (Figure $5 \mathrm{~d}$ ). In contrast, CAFs with LGALS1 shRNA migrated much slower, with a still visible gap after $24 \mathrm{~h}$ postwounding. To test for invasion, the CAF control and CAF LGALS1 shRNA cells were placed into transwell coated with Matrigel. After $24 \mathrm{~h}$, the cell number of the CAF LGALS1shRNA cells that invaded through the transwell was significantly reduced (Figure 5e, $P<0.001$ ) compared with parental CAF cells. These observations suggested that knock- 
down of galectin-1 in pancreatic CAFs can dramatically reduce cell migration and invasion capabilities.

\section{DISCUSSION}

PDACs typically have very exuberant stroma, which can make up to $90 \%$ of the tumor mass that surrounds the tumor cells, having important mechanistic roles in the progression of PDAC. In our study, we used microscopic macro-dissection to dissect the tissue areas with the highest neoplastic cellularity (including both PDAC epithelium and associated stroma), while excluding areas of non-neoplastic pancreatic acinar tissues and inflammatory infiltrates. Therefore the tissues we analyzed were primarily comprised of neoplastic cells and stromal cells. Although the mass spectrometry data may not provide us enough information to completely decode a signal from a heterogeneous population of cells, it allowed us to identify proteins with an overall abundance change in the tumor tissues. Such an approach has pros and cons, reflecting our emphasis on biomarker discovery and acquisition of information regarding tumor microenvironment. The subsequent IHC analysis permitted us to further examine a particular protein candidate for its distribution among different cell types and extracellular matrix (ECM). It is also notable that VLTS of pancreatic cancer are rare; and inclusion of only pathologically and clinically well-defined cases compromised the number of the specimens available for our initial proteomics discovery. Nonetheless, the protein profiling data was informative; and the IHC validation of selected protein candidates using expanded number of PDAC cases (independent cohort) with various overall survival times provided a diagonal confirmation on the selected targets.

The comparison of pancreatic tumor tissues of resectable PDAC patients with VLTS vs STS revealed a group of differentially expressed proteins associated with VLTS and STS patients, respectively, including PRELP and LGALS1 which were also evidenced in our previous work. ${ }^{13}$ The most enriched functional cluster of STS-associated proteins was cytoskeleton, which is a dynamic cellular structure that maintains cell shape, adhesion, motility, as well as intracellular trafficking and signaling. It is now well recognized that tumorigenesis is associated with altered cytoskeletal proteins and that these proteins clearly have a role in the metastatic process. ${ }^{30,31}$ We also noticed that several proteins associated with epithelial-mesenchymal transition, which has a pivotal role in the tumor progression, were upregulated in the STS compared with the VLTS, including laminin, integrin beta-1, smooth muscle actin and MMP2. Two classic mesenchymal markers (vimentin and fibronectin), however, did not display significantly different abundance between VLTS and STSwhich may be due to various factors and requires further confirmation.

The second enriched cluster for STS proteins was protein synthesis/ribonucleoprotein complex/RNA processing, reflecting the underlying active cellular biosynthesis that contributes to tumor growth. The third and common enriched cluster for both the STS- and VLTS-associated proteins was generation of precursor metabolites and energy. In proliferating cells, cellular metabolism provides both energy needed for maintaining homeostatic processes and the precursors for macromolecular biosynthesis needed for proliferation. Cancer cells have dysregulated cellular energy metabolism. More than 50 years ago, Warburg demonstrated that cancer cells had unusually high glycolytic activity even in aerobic condition (Warburg Effect). Today, it is generally regarded that cancer cells must rewire their metabolic programming in order to meet the demands of high energy and anabolic processes necessary for rapid growth. In our study, we observed that differential metabolism-related proteins (including metabolites, energy generating, glycolysis and TCA cycle) in the pancreatic cancer tissues were related to patient survival, suggesting that metabolic programming differs between pancreatic cancer patients with LTS and patients with STS. Our observation supports the notion that tumors display heterogeneity with regard to their metabolic reprogramming. ${ }^{32}$ Such metabolic reprogramming could be driven by both genetic (mutation) and non-genetic (tumor microenvironment) factors ${ }^{32}$ and could ultimately influence cancer survival.

Using IHC analysis on TMA, we demonstrated that the PRELP abundance level was statistically associated with longer survival. PRELP is a proteoglycan found in the ECM. It binds perlecan and collagens and may function as a basement membrane anchor. ${ }^{33}$ PRELP was found as one of the predictor gene signatures for brain tumors. ${ }^{34}$ Additionally, PRELP can act as a NF- $\mathrm{KB}$ inhibitor impairing osteoclastogenesis. ${ }^{35} \mathrm{NF}-\kappa \mathrm{B}$ is constitutively overexpressed in PDAC and its inhibition is a mandate for a new class of therapeutic drugs. Related to this observation, we also discovered that copines were overexpressed in the VLTS proteomic analysis. Although the functional role of these calcium-dependent membrane proteins is mostly unknown, copine- 1 has been identified as another NF- $\mathrm{KB}$ inhibitor. ${ }^{36}$ These combined observations suggest that repression of NF- $\mathrm{KB}$ could prolong survival in pancreatic cancer.

RPS8, a component of the ribosomal 40S subunit, was found to be inversely associated with survival in our study. Increased abundance of ribosomal genes, including RPS8, was previously observed in colorectal tumors and colon polyps compared with matched normal colonic mucosa. ${ }^{37}$ Studies also suggested that the abundance of RPS 8 could determine the susceptibility of pancreatic cancer cells to gemcitabine treatment. ${ }^{38}$ In the current study, we observed that higher abundance of RPS8 was associated with worse survival of pancreatic cancer. The combination of 'HighRPS8 and Low-PRELP' provided improved prognostic value over RPS8 or PRELP alone.

A third protein related to pancreatic cancer survival was galectin-1. Upregulation of galectin-1 has been documented for several tumor types in both the cancerous epithelial cells, as well as in the stroma adjacent to cancer cells. ${ }^{39}$ Galectin-1 
is a $\beta$-galactoside-binding protein that is involved in immunosuppression and angiogenesis. Recent study suggests that galectin-1 could bind to glycosylated VEGFR in endothelial cells to promote angiogenesis in the absence of VEGF. ${ }^{40}$ In the comparison of VLTS and STS of pancreatic cancer patients, we consistently observed the lower abundance of galectin-1 in tumor tissues of the VLTS patients. IHC analysis indicated that galectin- 1 was mainly detected in the CAFs but not in cancer epithelial cells. ${ }^{13}$ Knockdown of galectin-1 gene in CAF cells significantly reduced cell migration and invasion of CAFs, implicating its potential role in tumor microenvironment contributing to pancreatic cancer progression and metastasis. Inhibition of galectin-1 as a potential therapeutic target has been evaluated in breast, colon, ovary and prostate cancers. ${ }^{41}$ Our results further support that gelactin-1 could be a potent target for pancreatic cancer therapy.

Supplementary Information accompanies the paper on the Laboratory Investigation website (http://www.laboratoryinvestigation.org)

\section{ACKNOWLEDGMENTS}

We thank Dr Ralph H Hruban for his assistance with histopathology of the cases from Johns Hopkins. This study was supported in part with federal funds from the National Institutes of Health under grants K07CA116296, K25CA137222, R21CA161575, R21CA149772, R01CA180949 and private funds from Gene and Mary Ann Walters Pancreatic Cancer Foundation and Donald E Bocek Endowed Research and Development Award.

\section{DISCLOSURE/CONFLICT OF INTEREST}

The authors declare no conflict of interest.

1. Siegel R, Naishadham D, Jemal A. Cancer statistics, 2012. CA Cancer J Clin 2012;62:10-29.

2. Adham $M$, Jaeck $D$, Le $B J$, et al. Long-term survival (5-20 years) after pancreatectomy for pancreatic ductal adenocarcinoma: a series of 30 patients collected from 3 institutions. Pancreas 2008;37:352-357.

3. Bradley III EL. Long-term survival after pancreatoduodenectomy for ductal adenocarcinoma: the emperor has no clothes? Pancreas 2008:37:349-351.

4. Cleary SP, Gryfe R, Guindi M, et al. Prognostic factors in resected pancreatic adenocarcinoma: analysis of actual 5-year survivors. J Am Coll Surg 2004;198:722-731.

5. Conlon KC, Klimstra DS, Brennan MF. Long-term survival after curative resection for pancreatic ductal adenocarcinoma. Clinicopathologic analysis of 5-year survivors. Ann Surg 1996;223:273-279.

6. Han SS, Jang JY, Kim SW, et al. Analysis of long-term survivors after surgical resection for pancreatic cancer. Pancreas 2006;32:271-275.

7. Mosca F, Giulianotti PC, Balestracci $T$, et al. Long-term survival in pancreatic cancer: pylorus-preserving versus Whipple pancreatoduodenectomy. Surgery 1997;122:553-566.

8. Reddy S, Wolfgang $\mathrm{CL}$, Cameron JL, et al. Total pancreatectomy for pancreatic adenocarcinoma: evaluation of morbidity and long-term survival. Ann Surg 2009;250:282-287.

9. Riall TS, Cameron JL, Lillemoe KD, et al. Resected periampullary adenocarcinoma: 5-year survivors and their 6- to 10-year follow-up. Surgery 2006;140:764-772.

10. Schnelldorfer $T$, Ware $A L$, Sarr MG, et al. Long-term survival after pancreatoduodenectomy for pancreatic adenocarcinoma: is cure possible? Ann Surg 2008;247:456-462.

11. Shimada K, Sakamoto Y, Nara S, et al. Analysis of 5-year survivors after a macroscopic curative pancreatectomy for invasive ductal adenocarcinoma. World J Surg 2010;34:1908-1915.
12. Allen PJ. Pancreatic adenocarcinoma: putting a hump in survival. J Am Coll Surg 2007;205:S76-S80.

13. Chen R, Pan S, Ottenhof NA, et al. Stromal galectin-1 expression is associated with long-term survival in resectable pancreatic ductal adenocarcinoma. Cancer Biol Ther 2012;13:899-907.

14. Garcea G, Dennison AR, Pattenden CJ, et al. Survival following curative resection for pancreatic ductal adenocarcinoma. A systematic review of the literature. JOP 2008;9:99-132.

15. Withers SG, Aebersold R. Approaches to labeling and identification of active site residues in glycosidases. Protein Sci 1995;4:361-372.

16. luga $C$, Seicean $A$, lancu $C$, et al. Proteomic identification of potential prognostic biomarkers in resectable pancreatic ductal adenocarcinoma. Proteomics 2014;14:945-955.

17. Nguyen KA, Arensman M, Lay AR, et al. HOXB7 promotes invasion and predicts survival in pancreatic adenocarcinoma. Cancer 2013;119: 529-539.

18. Pan $S$, Chen R, Stevens $T$, et al. Proteomics portrait of archival lesions of chronic pancreatitis. PLoS One 2011;6:e27574.

19. Craig R, Beavis RC. TANDEM: matching proteins with tandem mass spectra. Bioinformatics 2004;20:1466-1467.

20. Keller A, Eng J, Zhang N, et al. A uniform proteomics MS/MS analysis platform utilizing open XML file formats. Mol Syst Biol 2005;1:2005.

21. Keller SR, Kitagawa K, Aebersold R, et al. Isolation and characterization of the 160,000 -Da phosphotyrosyl protein, a putative participant in insulin signaling. J Biol Chem 1991;266:12817-12820.

22. Nesvizhskii Al, Keller A, Kolker E, et al. A statistical model for identifying proteins by tandem mass spectrometry. Anal Chem 2003;75:4646-4658.

23. Huang dW, Sherman BT, Lempicki RA. Systematic and integrative analysis of large gene lists using DAVID bioinformatics resources. Nat Protoc 2009;4:44-57.

24. Brentnall TA, Lai LA, Coleman J, et al. Arousal of cancer-associated stroma: overexpression of palladin activates fibroblasts to promote tumor invasion. PLoS One 2012;7:e30219.

25. Chen R, Yi EC, Donohoe $S$, et al. Pancreatic cancer proteome: the proteins that underlie invasion, metastasis, and immunologic escape. Gastroenterology 2005;129:1187-1197.

26. Chen R, Brentnall TA, Pan $S$, et al. Quantitative proteomics analysis reveals that proteins differentially expressed in chronic pancreatitis are also frequently involved in pancreatic cancer. Mol Cell Proteomics 2007;6:1331-1342.

27. Pan $\mathrm{S}$, Chen R, Reimel BA, et al. Quantitative proteomics investigation of pancreatic intraepithelial neoplasia. Electrophoresis 2009;30: 1132-1144.

28. Dennis Jr. G, Sherman BT, Hosack DA, et al. DAVID: Database for Annotation, Visualization, and Integrated Discovery. Genome Biol 2003;4:3.

29. Tomsig JL, Creutz CE. Copines: a ubiquitous family of $\mathrm{Ca}(2+)$ dependent phospholipid-binding proteins. Cell Mol Life Sci 2002;59: 1467-1477.

30. Olson MF, Sahai E. The actin cytoskeleton in cancer cell motility. Clin Exp Metastasis 2009:26:273-287.

31. Yilmaz M, Christofori G. EMT, the cytoskeleton, and cancer cell invasion. Cancer Metastasis Rev 2009;28:15-33.

32. Cantor JR, Sabatini DM. Cancer cell metabolism: one hallmark, many faces. Cancer Discov 2012;2:881-898.

33. Bengtsson $E$, Morgelin $M$, Sasaki $T$, et al. The leucine-rich repeat protein PRELP binds perlecan and collagens and may function as a basement membrane anchor. J Biol Chem 2002;277: 15061-15068.

34. Castells X, Garcia-Gomez JM, Navarro A, et al. Automated brain tumor biopsy prediction using single-labeling CDNA microarrays-based gene expression profiling. Diagn Mol Pathol 2009;18:206-218.

35. Rucci N, Rufo A, Alamanou M, et al. The glycosaminoglycan-binding domain of PRELP acts as a cell type-specific NF-kappaB inhibitor that impairs osteoclastogenesis. J Cell Biol 2009;187:669-683.

36. Ramsey CS, Yeung F, Stoddard PB, et al. Copine-I represses NF-kappaB transcription by endoproteolysis of p65. Oncogene 2008;27: 3516-3526.

37. Pogue-Geile K, Geiser JR, Shu M, et al. Ribosomal protein genes are overexpressed in colorectal cancer: isolation of a CDNA clone encoding the human S3 ribosomal protein. Mol Cell Biol 1991;11:3842-3849. 
38. Toshimitsu $\mathrm{H}$, lizuka N, Yamamoto $\mathrm{K}$, et al. Molecular features linked to the growth-inhibitory effects of gemcitabine on human pancreatic cancer cells. Oncol Rep 2006;16:1285-1291.

39. Astorgues-Xerri L, Riveiro ME, Tijeras-Raballand A, et al. Unraveling galectin-1 as a novel therapeutic target for cancer. Cancer Treat Rev 2013;40:307-319.
40. Croci DO, Cerliani JP, Dalotto-Moreno T, et al. Glycosylationdependent lectin-receptor interactions preserve angiogenesis in anti-VEGF refractory tumors. Cell 2014;156:744-758.

41. Ito K, Stannard K, Gabutero E, et al. Galectin-1 as a potent target for cancer therapy: role in the tumor microenvironment. Cancer Metastasis Rev 2012;31:763-778. 